\title{
A PSAM-Based Estimator of Noise and Fading Statistics for Optimum Receivers of Free Space Optics Signals
}

\author{
Hassan Moradi ${ }^{*}$, Hazem H. Refai ${ }^{*}$, Peter G. LoPresti ${ }^{* *}$, Mohammed Atiquzzaman ${ }^{* * *}$ \\ "Electrical and Computer Engineering, University of Oklahoma, Tulsa, OK. \\ (Tel:918660-3243,e-mails: hmoradi@ou.edu, hazem@ou.edu) \\ ${ }^{* *}$ Electrical Engineering Department, University of Tulsa, Tulsa, OK. \\ (Tel:918631-3274,e-mail:pgl@ohm.ee.utulsa.edu) \\ ${ }^{* * *}$ Computer Science, University of Oklahoma, Norman, OK. \\ (Tel: 405 325-8077,email: atiq@ou.edu)
}

\begin{abstract}
Incoherent receivers of Free Space Optical (FSO) signals have no knowledge of instantaneous channel state. Thus, the receiver requires some information about the noise and fading statistics for a maximum likelihood (ML)-based optimal detection. Using pilot-aided symbols, we develop a simple multi slot averaging (MSA) estimation technique to approximate the values of parameters required at the incoherent detector. No channel state information (CSI) is available at the receiver side and this work will not be also trying to estimate it. But the estimation of noise and fading statistics will be practically investigated. We evaluate the bit error rate (BER) performance of FSO links with MSA estimation over both Gaussian and lognormal atmospheric turbulence fading (scintillation) channels. Numerical simulation will be completed to evaluate the estimation error of the MSA estimator. We will see that at signal to noise ratio $(\mathrm{SNR})=13 \mathrm{~dB}$, the performance loss of the Gaussian estimator improves from $3 \mathrm{~dB}$ to $0.4 \mathrm{~dB}$ when we increase the number of pilot symbols from 16 to 64 . This paper also presents the hardware design of the estimator using Xilinx system generator.
\end{abstract}

Keywords: FSO, Channel estimation, lognormal fading, noise statistics, optimal detection, estimation error.

\section{INTRODUCTION}

Free space optics communication is a cost-effective, high-bandwidth access technique, which has received increasing attention and achieved recent commercial success. However, long-distance outdoor FSO links are highly vulnerable to the degrading effects of atmospheric turbulence, i.e. fading or scintillation, which severely impair link performance. Atmospheric turbulence results from variations in the refractive index caused by inhomogeneties in temperature and changes in pressure. The most efficient and promising receivers of digital communication systems require information from the transmission channel. This is achieved using a channel estimation mechanism. There are two type of channel information: instantaneous fading coefficient and fading (and noise) statistics. The first one which refers to CSI in literature is not the focus of this paper. We consider the incoherent detection for the received FSO signal similar to the assumptions made in [1] and [2]. In both works, a detection of OOK (on-off keying) with constant fading intensity was investigated, where the receiver does not have nor explicitly attempts to estimate fading coefficient value. We investigate the known pilot symbols (bits) from the channel to measure the parameters required for optimal detection, thus providing the minimization of the performance loss due to estimation error.

Pilot symbol (PS) assisted (or aided) modulation (PSAM) techniques have been used for optimal-incoherent (direct) detection of OOK modulation in free space optics communication [3]. However, the incoherent detection can be realized without the use of pilot symbols (e.g. Manchester encoding). But they may have some disadvantages. The primary objective of this work is the design of a PSAM-based estimation system for FSO links in practice. This paper basically investigates the effect of noise and fading statistics estimation on the performance of optimal detection of FSO signals. The system model assumes both Gaussian and lognormal fading through the transmission channel. Consequently, the performance loss due to estimation error will be evaluated.

In this paper we first briefly analyze the effect of additive noise and atmosphere turbulence on the FSO link performance. Then the estimation procedure will be explained and presented. The effectiveness and performance of the estimation method will be investigated by a simulator designed for hardware implementation of the estimator.

Free-Space Laser Communication Technologies XXII, edited by Hamid Hemmati, Proc. of SPIE Vol. 7587, 758700 - @ 2010 SPIE · CCC code: $0277-786 X / 10 / \$ 18 \cdot$ doi: $10.1117 / 12.844906$ 


\section{FSO THROUGH ATMOSPHERE TURBULENCE}

We consider OOK modulation for transmitting information symbols. The on-condition corresponds to symbol '1', and the off-condition corresponds to symbol ' 0 '. At the end of a symbol period, the receiver's output is compared to a set threshold value $I_{D}$, and a decision on the transmitted symbol is made. In this part we provide the system model of a FSO fading channel where the additive noise is also added to the transmitted signal.

\subsection{OOK Encoding in Gaussian Channel}

Assuming perfect noise parameters are available at the receiver side, an optimum value of BER on the basis of maximum likelihood is calculated as [4]

$$
B E R=\frac{1}{2} \operatorname{erfc}\left(\frac{I_{1}-I_{0}}{\sqrt{2}\left(\sigma_{1}+\sigma_{0}\right)}\right)
$$

where $I_{1}$ and $I_{0}$ are the means of the generated currents at the photodetector, and $\sigma_{1}$ and $\sigma_{0}$ are the standard deviations for symbols ' 1 ' and ' 0 ', respectively. In this paper we call these four parameters as noise statistics. The threshold current considered as the decision criterion at the receiver will be given by

$$
I_{D}=\frac{\sigma_{0} I_{1}+\sigma_{1} I_{0}}{\sigma_{0}+\sigma_{1}}
$$

It can be easily inferred that errors occur if the receiver's output current $i_{p}<I_{D}$ for symbol ' 1 ' or if $i_{p}>I_{D}$ for symbol ' 0 ' is sent. There is a relation among these noise parameters as [5], [6]

$$
\begin{gathered}
\sigma_{1}^{2}=2 q B_{e q} I_{1}+\sigma_{t}^{2} \\
\sigma_{0}^{2}=2 q B_{e q} I_{0}+\sigma_{t}^{2}
\end{gathered}
$$

where $q$ is electron charge constant $(\mathrm{C}), B_{e q}$ is noise equivalent bandwidth (NEBW) (Hz), and $\sigma_{t}$ is the standard deviation of the Gaussian noise current due to thermal noise at the photodiode receiver. The averaged SNR can be expressed by

$$
S N R=\frac{\left(I_{1}-I_{0}\right)^{2}}{\left(\sigma_{1}+\sigma_{0}\right)^{2}}
$$

We use this expression as the averaged SNR in the analysis.

\subsection{Manchester Encoding in Gaussian Channel}

A common representation of encoding is the Manchester method. The system uses pulse signaling, where the pulse time is one half the bit interval, thus a shorter time interval than for the OOK system [4]. This is considered as a returnto-zero (RZ) transmission format. To ease the performance comparison between these two modulations, the BER can be rewritten as

$$
B E R_{m}=\frac{1}{2} \operatorname{erfc}\left(\frac{I_{1}-I_{0}}{2 \sqrt{\left(\sigma_{1}^{2}+\sigma_{0}^{2}\right)}}\right)
$$

Manchester pulsed coding is a popular way to bypass the need to estimate the receiver's required parameters. Theoretically, it provides close performance to OOK; however, it does not require channel estimation, nor does it meet estimation error. Rather it encounters a duplication of the available bandwidth and a more complex apertures design. Consequently it results in a more complex optical transmitter and receiver design in high data-rate transmission.

\subsection{Atmosphere Turbulence Fading}

In free space optics, the interaction between light and atmosphere manifests its effects through various phenomena, namely attenuation, absorption, scattering, and scintillation. This occurs with the introduction of random, time-varying amplitude gain and a signal phase shift. The channel effect must be mitigated in order to obtain a high performance coherent detection of a modulated optical signal. When incoherent detection of IM/DD is used (e.g. OOK), the optical signal model is real-valued, and there is no need for investigation of channel phase.

For OOK intensity modulation, the transmitted signal $s(t) \in\{0,1\}$ is passing through a fading channel thus the received electrical signal is then given by

$$
i_{p}(t)=h(t) s(t)+i_{n}(t)
$$


where $h(t)$ is the normalized channel fading intensity due to atmospheric turbulence and $i_{n}(t)$ is total additive noise, described by $N\left(\mu_{n}, \sigma_{n}\right)$ where $\mu_{n}$ and $\sigma_{n}$ are the mean and standard deviation the noise current, respectively. For simplicity we neglect time $(t)$ in analysis. Since various noise currents are not correlated, $\sigma_{n}$ can be expressed as the sum of Gaussian shot noise and thermal noise [5], [6]. Without loss of generality, we can separate the total atmospheric field attenuation into two components, $h=h_{1} h_{2}$. A non-random component $h_{1}$ is the result of the irretrievable power loss from absorption and scattering and a random component $h_{2}$, which results from turbulence-induced fading [7]. Random component $h_{2}$ and is the focus of this work when talking about channel coefficient or fading.

A number of analytical efforts for FSO channel modeling have been previously carried out in order to describe turbulence-induced degradation and intensity fluctuations. Interested readers can refer to one of the following references: studies of channel loss and the attenuations due to fog, rain, snow and scintillation [8], lognormal modeling of atmospheric turbulence-fading [2], the effect of strong turbulence fading along on the BER of FSO link [9], and lognormal fading channel due to scintillation has been proposed in [10].

\subsection{Lognormal Turbulence Fading Model} [6]

The fading channel coefficient, which models the channel in terms of the transmit and receive intensities, is given by

$$
h=\frac{I}{I_{m}}=\exp (2 X)
$$

where $\mu_{x}$ is the ensemble average of log-amplitude $X, I_{m}$ is the signal light intensity without turbulence at the transmitter, $I$ is the signal light intensity with turbulence at the receiver, and $X$ is identically distributed normal random variable with mean $\mu_{x}$ and standard deviation $\sigma_{x}$ :

$$
f(X)=\frac{1}{\sqrt{2 \pi} \sigma_{x}} \exp \left(-\frac{\left(X-\mu_{x}\right)^{2}}{2 \sigma_{x}^{2}}\right)
$$

Consider the propagation of light through a large number of atmospheric elements, each causing an independent, yet identically distributed, phase delay and scattering. The marginal distribution of the log-amplitude is Gaussian and can be expressed by a distribution function like (8). Therefore, the distribution of light intensity fading induced by turbulence is a lognormal distribution, which is expressed by

$$
f_{x}(h)=\frac{1}{\sqrt{8 \pi} h \sigma_{x}} \exp \left(-\frac{\left[\ln (h)+2 \sigma_{x}^{2}\right]^{2}}{8 \sigma_{x}^{2}}\right)
$$

where we assumed $E[h]=\mu_{h}=1$. It considers scintillation scattering, which is a representation of the constant total power through fading. Doing so requires the choice of $\mu_{x}=-\sigma_{x}^{2}$, which is posed in [7]. Thus, the variance will be equal to

$$
\sigma_{I}^{2}=e^{4 \sigma_{x}^{2}}-1
$$

This parameter is so called scintillation index, S.I. It is shown in [1] that for propagation distances of a kilometer, $\sigma_{x}$ varies from $10^{-2}$ to 1 for different conditions of scintillation turbulence. Further details about $\sigma_{x}$ measurements of the vertical profile can be found in [8], [11], [12] and [13]. Based on the variation range of values for $\sigma_{x}$, values for S.I. may be in the range $4 * 10^{-4}-54$. Typical S.I. values of $0.1-1.0$ appear in the literature. Low S.I. corresponds to less scattering, and a higher S.I. reflects more detrimental fading.

\subsection{Optimal Detection}

Transmitted symbols are decoded from the received data on the basis of the maximum likelihood of possible code words. An expression of the ML-based optimum decision threshold for a non-fading Gaussian channel model was obtained in Section 2. Remembering (2), the optimum decision metric for the received current $i_{p}$ may be expressed as

$$
\underset{0}{i_{p}} \underset{0}{\stackrel{\sigma_{0} I_{1}+\sigma_{1} I_{0}}{\sigma_{0}+\sigma_{1}}}
$$


The above detection rule is achieved based on a maximum likelihood criterion. For such a channel, the likelihood factorizes into a product of likelihoods for each symbol. For detection purposes, we can rewrite the likelihood function for a symbol to be ' 1 ' versus ' 0 ' as the conditional probabilities. The ML decision rule is

$$
\Lambda\left(i_{p}\right)=\frac{p\left(i_{p} \mid 1\right)}{p\left(i_{p} \mid 0\right)} \underset{0}{1}
$$

where $i_{p}$ is the photodetector's output current as the receive signal. But if the $i_{p}$ is assumed to be the output of a fading channel, the conditional probability density of level ' 0 ' can be expressed as

$$
p\left(i_{p} \mid 0\right)=\frac{1}{\sqrt{2 \pi} \sigma_{0}} \exp \left[-\frac{\left(i_{p}-I_{0}\right)^{2}}{2 \sigma_{0}^{2}}\right]
$$

which is the same as Gaussian channel because the fading does not affect the symbol ' 0 '. But the probability density of level ' 1 ' is affected by fading. In this case, $i_{p}$ is expressed by

$$
i_{p}=2 R h P_{t}+i_{n}
$$

where $i_{n}$ is the Gaussian noise signal with mean $I_{0}, P_{t}$ is the average transmitted optical power, $h$ is the fading coefficient and $R$ is receiver's responsivity (i.e. optical to electrical efficiency). We can conclude that $I_{1}-I_{0}=2 R P_{t} h^{1}$, however for simplicity we can assume $2 P_{t} R=1$. The conditional probability $p\left(i_{p} \mid 1\right)$ is given by

$$
p\left(i_{p} \mid 1\right)=\frac{1}{\sqrt{2 \pi} \sigma_{1}} \int_{0}^{\infty} f_{I}(h) \exp \left[-\frac{\left(i_{p}-I_{o}-2 R P_{t} h\right)^{2}}{2 \sigma_{1}^{2}}\right] d h
$$

The simplest expression for likelihood ratio then may be expressed as

$$
\Lambda\left(i_{p}\right)=\frac{\sigma_{0}}{\sigma_{1}} \int_{0}^{\infty} f_{I}(h) \exp \left[-\frac{\left(i_{p}-I_{o}-2 R P_{t} h\right)^{2}}{2 \sigma_{1}^{2}}-\frac{\left(i_{p}-I_{o}\right)^{2}}{2 \sigma_{0}^{2}}\right] d h
$$

The threshold value is the solution of equation $\Lambda\left(I_{D}\right)=1$ for $I_{D}$. However, the decision rule for any received signal $i_{p}$ will be given by

$$
\frac{\sigma_{0}}{\sigma_{1}} \int_{0}^{\infty} f_{I}(h) \exp \left[-\frac{\left(i_{p}-I_{o}-2 R P_{t} h\right)^{2}}{2 \sigma_{1}^{2}}-\frac{\left(i_{p}-I_{o}\right)^{2}}{2 \sigma_{0}^{2}}\right] d h{ }_{<}^{>}
$$

where $f_{I}(h)$ is the probability distribution function (PDF) of the fading defined by (9) as a lognormal channel. This type of detection is called symbol-by-symbol detection [1].

\section{CHANNEL ESTIMATION}

The fundamental objective of channel estimation in this paper is to measure (or estimate) the receiver parameter values $\left(I_{D}\right.$, expressed in Eq. (2)) from only the received OOK signal, (i.e. by means of pilot sequences) in order to provide an optimal decision. In fact, the statistics of channel (but not instantaneous channel coefficient, CSI) can be estimated from the received signal if the effect of the channel on the data symbols can be evaluated. This is possible if the known (pilot) symbols are periodically available in the received packets. The focus of this section is the description of a pilot symbol-based channel estimation method, sometimes so called Data-Aided (DA) [14] or nonblind [15].

\subsection{Feasibility of Channel Estimation for FSO links}

In terms of FSO communication, PSAM technique is proposed [3], where Zhu et al. propose two schemes to mitigate correlated channel fading. A signal is transmitted through a simple fading channel in IM/DD links under the assumption that the fading correlation properties are known and the instantaneous fading state is unknown. However the application of PSAM in this paper is completely different than the work by Zhu et al. We use PSAM for estimating the fading and noise statistics.

The time-variance property of fading channels is one of the major factors that make channel estimation more complicated. Fortunately, this property is relatively slow in FSO applications when compared with symbol period, so the

\footnotetext{
${ }^{1}$ - For non-fading channel equation $I_{1}-I_{0}=2 R P_{t}$ is valid.
} 
channel can be repeatedly estimated in periods of time where time-invariance can be assured. As mentioned in [9], the fluctuation frequency due to scintillation on the received signal amplitude is a value between 0.01 and $200 \mathrm{~Hz}$. We define this parameter as scintillation frequency. This figure is not comparable to the short symbol intervals in FSO link. Hence, the channel is assumed to be time-invariant over a symbol interval as a given observation.

\subsection{Pilot Modulation Structure}

Like the general form of the serial PSAM, the resulting frame structure is shown in Fig. 1. The symbols are formatted into frames of length $M$ slots in which the value of pilot symbols are known. Each slot includes both control and data information parts. Control information includes defined pilot symbols for channel estimation and a reserved filed for other aplications. Each frame of length $T_{f}$ split into $M$ slots; each of length $T_{s}$, corresponds to one estimation period.

Parameter $k$, bit rate factor, in Fig. 1 determines the total number of symbols per slot. This is useful for deploying a variable rate procedure for FSO link which is out of the scope of this paper. With this range of $k$, the link can cover a range of $10^{3} \mathrm{Kbps}$ to1280 Mbps of bit rate, including both data bits and control (pilot) symbols. Neglecting the Reserve field, the exact value for $N_{D}$ will be then given by

$$
N_{D}=2^{10+k}-N_{P} ; k=0, \ldots, 7
$$

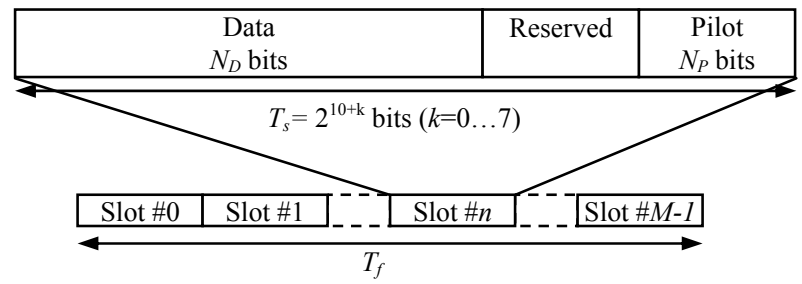

Fig. 1: Our practical frame structure for FSO packets. This figure shows the structure of one optical frame as the physical channel frame which consists of $M$ slots.

\subsection{Estimation Procedure}

The knowledge of noise and fading statistics including $\sigma_{1}, \sigma_{0}, I_{1}, I_{0}$ and $\sigma_{x}$ are estimated by channel estimation. Using Temporal Averaging (TA), the instantaneous estimated value for $I_{0}$ of a symbol ' 0 ' in the pilot symbols can be measured as

$$
\hat{I}_{0}^{e}(n, m)=\frac{1}{T_{b}} \int_{0}^{T_{b}} r_{t}(0 ; n, m) d t
$$

where $r_{t}(0 ; n, m)$, is the received signal current for $m$-th ' 0 ' symbol in the $n$-th slot and $T_{b}$ is the symbol interval equal to $1 /$ bitrate. $^{2}$ The detector receives completely noise for symbol ' 0 ' in OOK modulation. Hence, we can readily measure the instantaneous standard deviation of the noise current for this symbol as

$$
\hat{\sigma}_{0}^{e}(n, m)=\sqrt{\left(\frac{1}{T_{b}} \int_{0}^{T_{b}} r_{t}(0 ; n, m) d t-\hat{I}_{0}^{e}(n, m)\right)^{2}}
$$

If we assume a relatively high value for fluctuation (scintillation) frequency due to scintillation, a multi slot averaging (MSA) channel estimation can be employed. Based on this case, the performance of channel estimation improves if the channel estimation observation interval is extended to more than one slot and a larger number of pilot symbols are involved. Averaging is performed on a number of sequential slots. This may basically increases the complexity of the receiver in practice in terms of memory usage and processing delay. Thus a tradeoff exists between the estimation accuracy and the hardware complexity. Considering equal numbers for symbols ' 0 ' and ' 1 ' in the pilot field of each slot, the estimation of $I_{0}, \widetilde{I}_{0}^{e}$, for the $n$-th slot is performed using pilot symbols belonging to the $2 K+1$ slots according to

$$
\widetilde{I}_{0}^{e}(n)=\frac{2}{T_{b}(2 K+1) N_{P}} \sum_{i=-K}^{K} \sum_{m=0}^{N_{P} / 2-1} \int_{0}^{T_{b}} r_{t}(0 ; n+i, m) d t
$$

2- A same expression can be derived for bit ' 1 '. 
Similarly, the estimated mean square value of the noise current for symbol ' 0 ', $\widetilde{\sigma}_{0}^{e}$ is given by

$$
\tilde{\sigma}_{0}^{e}(n)=\sqrt{\frac{2}{T_{b}(2 K+1) N_{P}} \sum_{n=-K}^{K} \sum_{m=0}^{N_{P} / 2-1}\left(\int_{0}^{T_{b}} r_{t}(0 ; n, m) d t-\widetilde{I}_{0}^{e}(n, m)\right)^{2}}
$$

Since we assumed $E[h]=1$, the estimated value of $I_{1}, \widetilde{I}_{1}^{e}$, for the $n$-th slot is performed by

$$
\widetilde{I}_{1}^{e}(n)=\frac{2}{T_{b}(2 K+1) N_{P}} \sum_{i=-K}^{K} \sum_{m=0}^{N_{P} / 2-1} \int_{0}^{T_{b}} r_{t}(1 ; n+i, m) d t=2 R P_{t}+\widetilde{I}_{0}^{e}(n)
$$

We can use Eq. (3) to calculate the estimated standard deviation of noise current for symbol ' 1 ' when the channel imposes a fading effect on the signal. In this case $\sigma_{1}^{2}=2 q B_{e q}\left(I_{o}+2 R P_{t} h\right)+\sigma_{t}^{2}$ which is itself a random variable due to $h$. But it can be shown that the transmitted power has a very little effect on the performance. Thus even for large values of the transmitted power, we can assume that $\sigma_{1} \approx \sigma_{0}$. This means that measuring only three required noise parameters for the optimal receiver can provide estimation for the last one. Therefore, with these parameters, Eq. (2) is applicable to an optimal receiver. Fig. 2 summarizes the receiver block diagram of the described optimal receiver for FSO communication. Note that to estimate the required parameters for the $n$-th slot in the MSA method, slots from $n$ - $K$ to $n+K$ are used and averaged, as shown in Fig. 2a. In other words, $K$ next slots and $K$ previous slots are required to estimate the $n$-th slot.
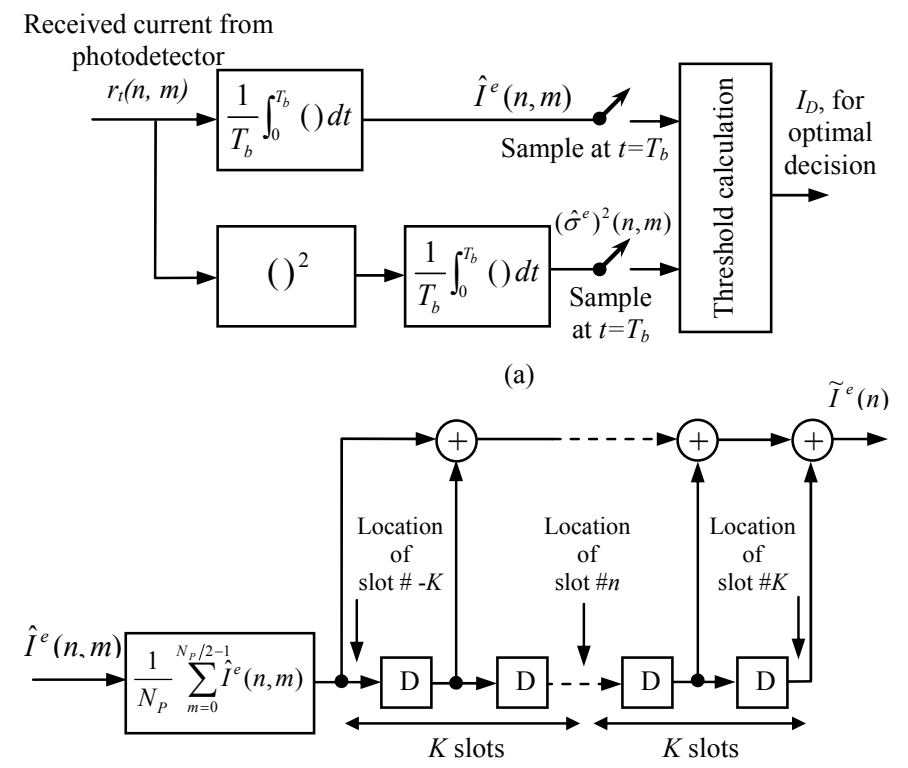

(b)

Fig. 2: Channel estimation diagrams for the noise statistics. (a) the diagram for calculating threshold current $I_{D}$ for optimal decision and (b) MSA channel estimation for $n$-th slot as a part of "Threshold calculation" block in (a). This figure shows estimating diagram for calculating the average values of the currents. Because of the similarity, the diagram for estimating the variances was not shown. The blocks, noted ' $\mathrm{D}$ ', represent the delay tabs between slots. A number of $2 K+1$ slots (and so with $2 K$ delay taps) is used for each slot to be estimated in a MSA.

Assuming that turbulence-induced fading coefficients at different time are uncorrelated, the statistics of a received symbol ' 1 ' is equal to

and from (10)

$$
\mu_{p, 1}=\mu_{I}+I_{0}=2 R P_{t}+I_{0}
$$

$$
\sigma_{p, 1}^{2}=\sigma_{I}^{2}+\sigma_{1}^{2}=e^{4 \sigma_{x}^{2}}+\sigma_{0}^{2}-1
$$

The estimated value of fading statistics then will be calculated from (25) by

$$
\hat{\sigma}_{x} \approx \frac{1}{2} \sqrt{\ln \left[\hat{\sigma}_{p, 1}^{2}-\hat{\sigma}_{0}^{2}+1\right]}
$$


where $\hat{\sigma}_{p, 1}$ is the estimated value of standard deviation for received symbols ' 1 ', using the pilot signal.

\section{SIMULATION AND ANALYSIS}

This section presents simulation results for the BER performance of FSO links based on some various ranges of the parameters effective for optimal detection. We will investigate the effectiveness of proposed estimation method for noise and fading statistics based on a FSO Lognormal channel. The BER performance realization in this paper are demonstrated when no error control coding is applied. However an effective coding mechanism may be employed to retrieve the corrupted symbols [16]. The Gaussian noise is added to the bit stream independently for any bit interval, and the lognormal channel effect described based on (9). Likewise, the fading includes only the scintillation effect and changes independently for any bit interval with a very small scintillation frequency. We assume no inter-symbol interference and with constant transmit power, SNR changes by variations in the noise power via parameter $\sigma_{0}$. An equal number of ' 1 ' and ' 0 ' symbols are assumed in each pilot field.

\subsection{Accuracy and Tracking Capability in a non-fading Channel}

Using a higher number of pilot symbols provides more accuracy for the estimation of noise and fading statistics resulting more tracking capabilities and, consequently, better performance of the receiver and MSA. However, the number of pilot symbols $N_{p}$, represents a trade-off between BER performance and energy and bandwidth in unnecessary pilot symbols.

Fig. 3 shows BER tracking ability for the proposed MSA estimation in a Gaussian channel (no fading), where only one slot $(K=0)$ is involved in each estimation period. The diagram compares the tracking ability of MSA estimation to the optimal BER that the detector can provide in the best case. Therefore, it is expected that ideal estimation offers a better performance (less BER) than MSA estimation. The detection is done based on a symbol-by-symbol detection in (11). To clarify the accuracy of estimating noise statistics, the normalized estimation error $\varepsilon_{e}=\left|\hat{P}_{e}-P_{e}\right| / P_{e}$ is presented in Fig. 4, where $\hat{P}_{e}$ and $P_{e}$ are the delivered BERs from detections based on estimated parameters and real values, respectively. $\varepsilon_{e}$ varies ranging from 0.01 to 100 for different values of applied $N_{p}$ and SNRs. Fig. 3 shows that when $N_{p-}$ $=64$, the performance loss of the estimator for a SNR ranging from $1 \mathrm{~dB}$ to $13 \mathrm{~dB}$ is just $0.4 \mathrm{~dB}$ in the worst case $(\mathrm{SNR}=13 \mathrm{~dB})$. At this point the normalized estimation error is 1 which means that the delivered BER is two times that from best optimal detection. Also, the receiver works much better than when using a pilot $N_{p}=16$, which causes performance loss of $3 \mathrm{~dB}$ and an estimation error 100 at $\mathrm{SNR}=13 \mathrm{~dB}$.

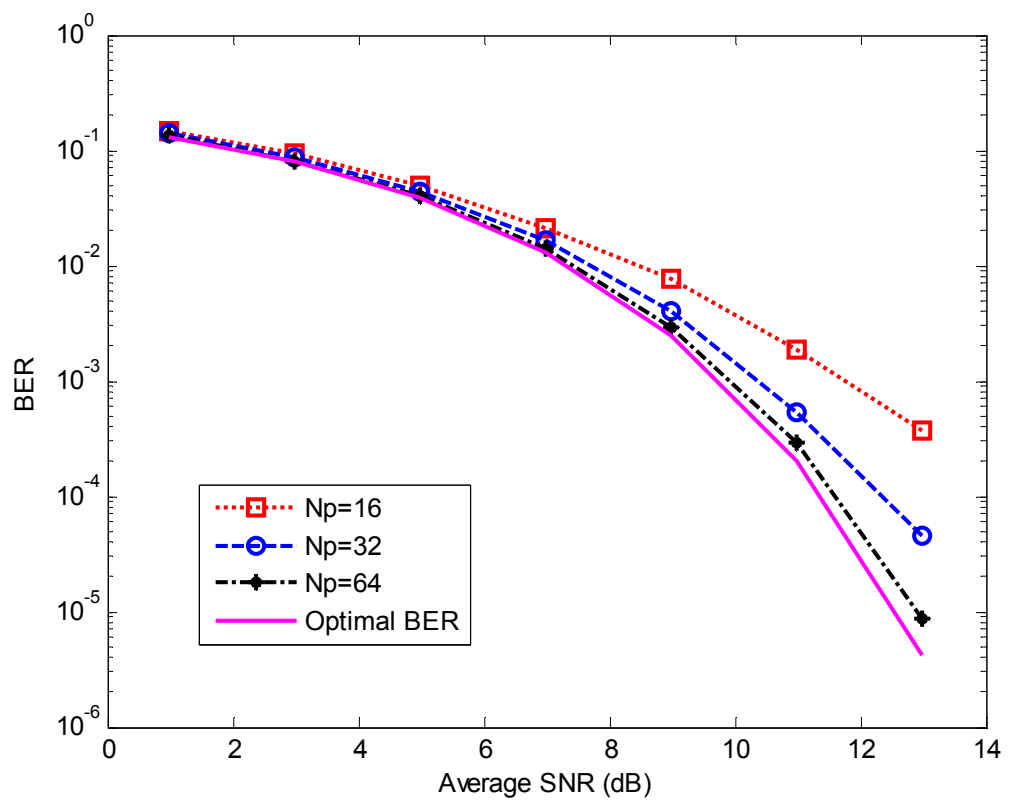

Fig. 3: The accuracy of MSA estimation for a FSO link in a Gaussian channel (no fading). BER vs. average received SNR, for different values of $N_{p} . K=0$. 


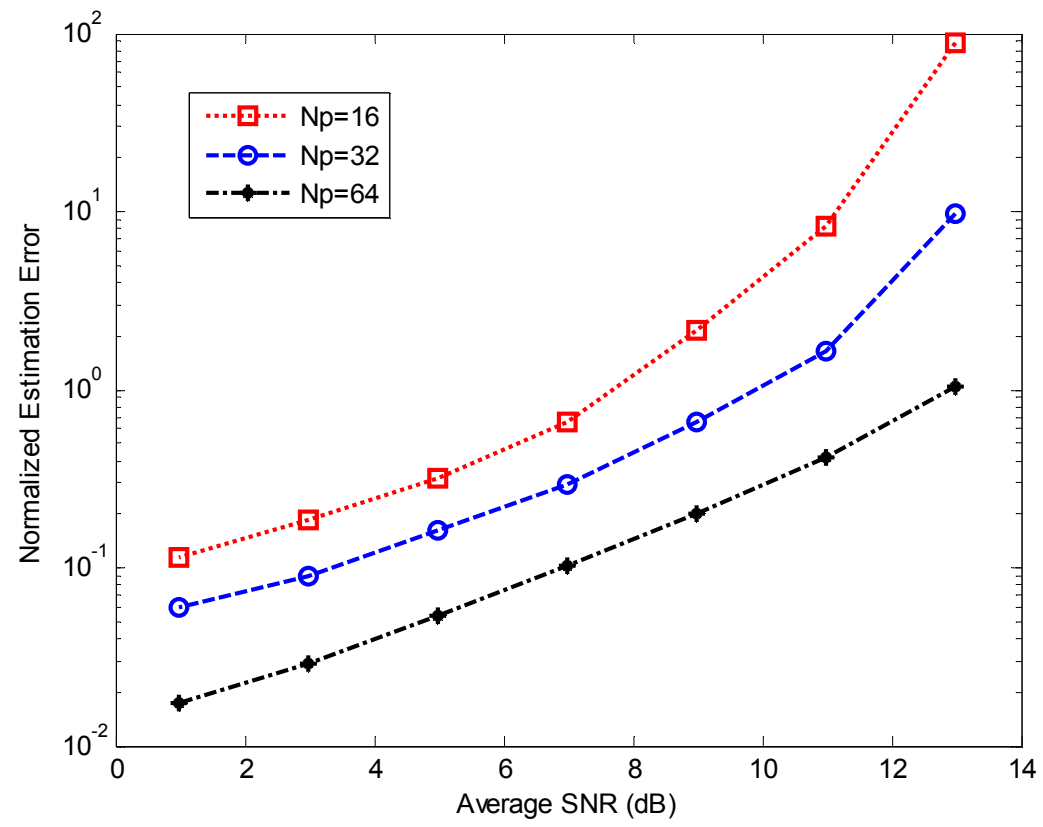

Fig. 4: The normalized estimation error of BER using the MSA estimator for a typical Gaussian channel. $K=0$.

\subsection{Accuracy and Tracking Capability in a Fading Channel}

We evaluated the variation of turbulence conditions of the lognormal fading on the MSA performance. This result is illustrated in Fig. 5 where it shows the BER tracking performance of the MSA method over a turbulence channel with three different values of $\sigma_{x}=0.4,0.2$ and 0.1 . The number of pilot symbols is chosen as a value of $N_{p}=64$ and we also chose $K=1$ - three slots involving for estimation of each slot. The detection is again done based on a symbol-by-symbol detection in (17). To perform a comparison, we also present the optimum detection when the receiver knows the exact values of noise and fading statistics. The normalized estimation error is also plotted in Fig. 6. It is observed that our proposed method of MSA channel estimation of optimal detection gives excellent matches to the best cases. Fig. 5 shows that the performance loss of the estimator working for a fading with $\sigma_{x}=0.1$ and a SNR ranging from $1 \mathrm{~dB}$ to $17 \mathrm{~dB}$ is just $0.5 \mathrm{~dB}$ in the worst case (at $\mathrm{SNR}=17 \mathrm{~dB}$ ). Although we have increased the number of averaged slots from $K=0$ at Gaussian channel to $K=3$ at lognormal channel, we may conclude that the effectiveness of the MSA method in Gaussian channel is better than lognormal.

\subsection{Implementation}

FSO links work with high data transmission rates and the detector should be implemented in hardware. The channel estimator block can be easily implemented by Xilinx System Generator tool installed in MATLAB. It is a plug-in to Simulink for developing high-performance Xilinx-based DSP systems. We have used version 8.2.02 of this tool with Virtex-5 FPGA development kit. Fig. 7 shows a snapshot of the receiver design in System Generator. We used this design to simulate the performance of our proposed estimator in Figs. 3 to 6 . The first block measures the mean parameters $I_{0}$ and $I_{1}$. It pulls out the pilot signals from the received packet based on Fig. 1. The second block is for variance estimation parameters $\sigma_{1}, \sigma_{0}$ and $\sigma_{x}$. The "Id calculator" block is used for calculating the threshold current $I_{D}$ in Eq. (2) while the detection block decides the received data is a 1 or 0 . The total delay of the receiver is only 84 samples which are due to the CORDIC blocks of system generator. 


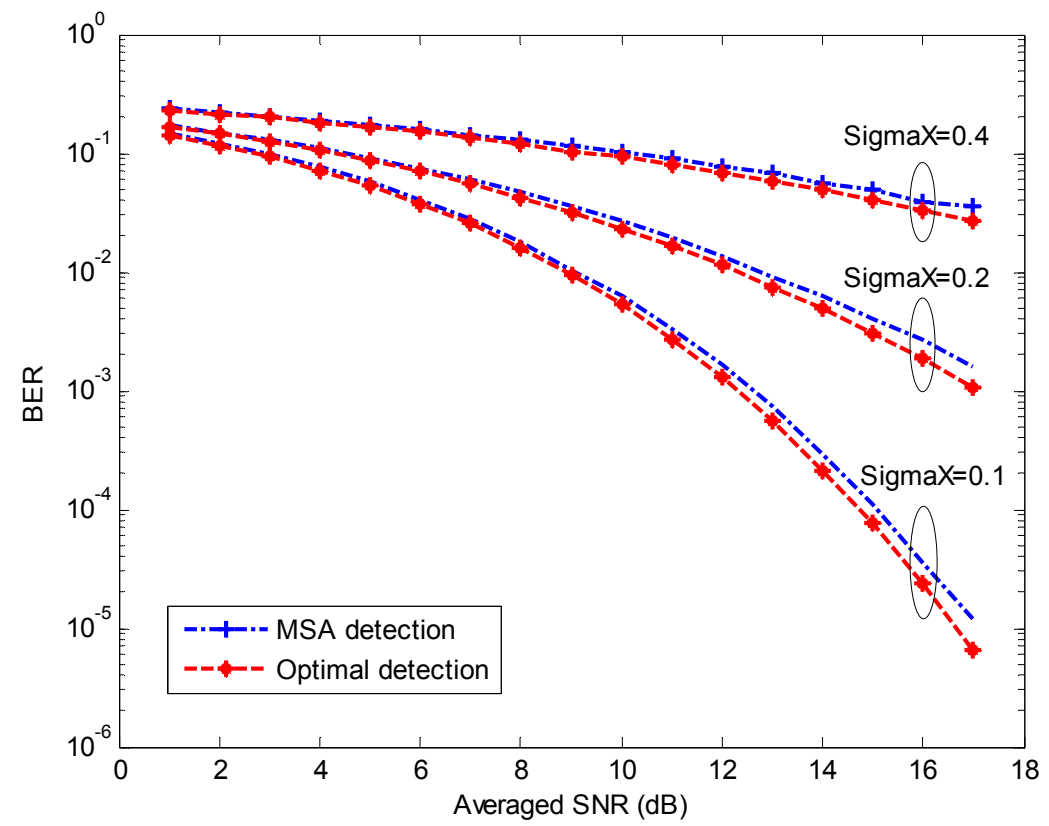

Fig. 5: The accuracy of MSA estimation for a FSO link in a lognormal channel. BER vs. average received SNR, for different value of fading intensity $\sigma_{x}$ based on a symbol-by-symbol detection. Case "Optimal detection" refers to the case that the receiver completely and ideally knows the values of the noise and fading statistics parameters. $K=1$ ( 3 slots) and $N_{p}=64$.

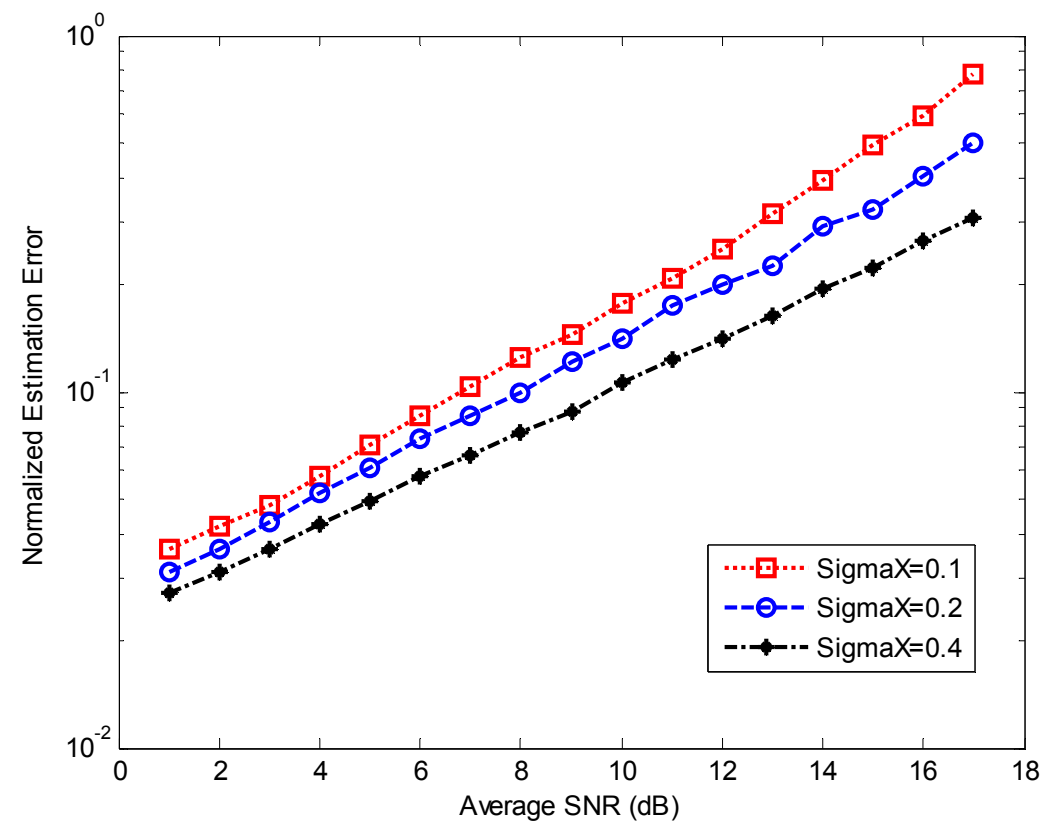

Fig. 6: The normalized estimation error when an MSA estimator is applied on a FSO link in a lognormal channel. Degradation in BER performance vs. average received SNR, for different value of fading intensity $\sigma_{x}$ based on a symbol-by-symbol detection. $K=3$ and $N_{p}=64$. 


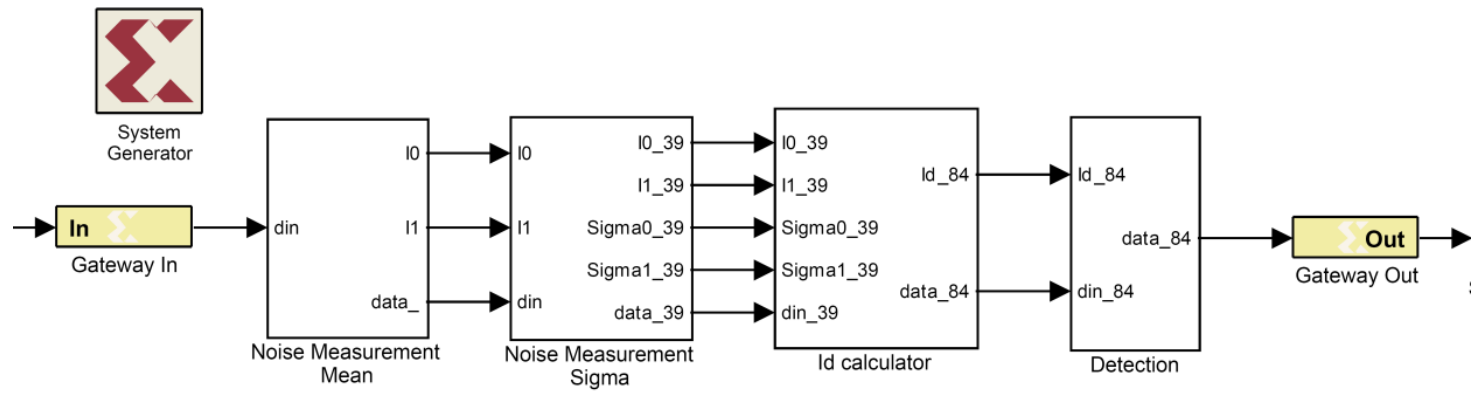

Fig. 7: A view of the receiver hardware design in Xilinx system generator.

\section{CONCLUSIONS}

The multi slot averaging (MSA) method was applied to provide a practical design for optimal detection of FSO signals. Frame structure was designed, and simulations were performed to evaluate the method performance in various cases. The results demonstrate that the MSA method can provide an uncompromising measurement for both Gaussian noise and with/without lognormal fading channel. MSA can mitigate the channel fading if we increase the number of pilot symbols to 64 symbols and the number of slots to 3 . In this case the performance loss will be equal to $0.5 \mathrm{~dB}$ at $\mathrm{SNR}=17 \mathrm{~dB}$ and $\sigma_{x}=0.1$. All of the simulation measurements and results are done in a hardware design by Xilinx system generator utilizing a Virtex-5 FPGA.

\section{ACKNOWLEDGEMENTS}

This work is funded by NSF grant number NSF-ECCS 0725801. The first author thanks Dr. Anjan K. Ghosh for helpful notes through the Free Space Optics course.

\section{REFERENCES}

[1] Zhu, X., and Kahn, J. M., Free-Space Optical Communication Through Atmospheric Turbulence Channels, IEEE Trans. Comm., Vol. 50, No. 8, pp. 1293-1300, Aug. 2002.

[2] Riediger, M. L. B. , Schober, R., and Lampe, L., Decision-Feedback Detection for Free-Space Optical Communications, Proceedings of the IEEE VTC-2007, pp. 1193-1197, 2007.

[3] Zhu, X., and Kahn, J. M., Pilot-Symbol Assisted Modulation for Correlated Turbulent Free-Space Optical Channels, Proceedings of the SPIE, Vol. 4489, pp. 138-145, 2002.

[4] Gagliardi, R. M., and Karp, S., Optical Communications, $2^{\text {nd }}$ edition, John Wiley \& Sons, Inc., 1995.

[5] Keiser, G., Optical Communications Essentials, McGraw-Hill, 2003.

[6] Aviv, G. D., Laser Space Communications, Artech House, 2006.

[7] Wilson, S. G., Brandt-Pearce, M., Cao, Q., and Baedke, M., Optical Repetition MIMO Transmission With Multipulse PPM , IEEE Journal on Selected Areas in Comm., Vol. 23, No. 9, Sept. 2005.

[8] Navidpour, S. M., Uysal, M., and Kavehrad, M., BER Performance of Free-Space Optical Transmission with Spatial Diversity, IEEE Trans. Wireless Comm., Vol. 6, No. 8, pp. 2813-2819, Aug. 2007.

[9] Muhammad, S. S., Kohldorfer, P., and Leitgeb, E., Channel Modeling for Terrestrial Free Space Optical Links, Proceedings of the ICTON'05, pp. 407-410, 2005.

[10] Wilson, S. G., Brandt-Pearce, M., Cao, Q., and Baedke, M., Optical Repetition MIMO Transmission With Multipulse PPM , IEEE Journal on Selected Areas in Comm., Vol. 23, No. 9, Sept. 2005.

[11] Zhu, X., and Kahn, J. M., Markov Chain Model in Maximum-Likelihood Sequence Detection for Free-Space Optical Communication through Atmospheric Turbulence Channels, IEEE Trans. Commun., vol.51, no.3, pp. 509-516, Mar.2003.

[12] Zilberman, A., Kopeika, N. S., and Sorani, Y., Laser Beam Widening as a Function of Elevation in the Atmosphere for Horizontal Propagation, Proceedings of the SPIE, Vol. 4376, pp. 177-188, 2001.

[13] Karp, S., Gagliardi, R. M., Moran, S. E, and Stotts, L. B., Optical Channels, Plenum Press, 1988.

[14] Juntti, M. J., and Latva-aho, M., Multiuser Receivers for CDMA Systems in Rayleigh Fading Channels, IEEE Trans. Vehicular Tech., Vol. 49, No. 3, pp. 885-899, May 2000.

[15] Boss, D., Kammeyer, K. D., and Petermann, T., Is Blind Channel Estimation Feasible in Mobile Communication Systems? A Study Based on GSM, IEEE Journal on Selected Areas in Comm., Vol. 16, No. 8, Oct. 1998.

[16] Kiasaleh, K., Hybrid ARQ Receiver for Packet Communications over Free-Space Optical Channels , Proceedings of the SPIE, Vol. 6304, pp. 630407-1-12, 2006. 